\title{
Carbonatos pedogénicos para el tratamiento del drenaje ácido de mina (DAM). Experimentos de laboratorio
}

\section{Pedogenic carbonates for the treatment of acid mine drainage (AMD). Laboratory experiments}

Maria Yazmin Rivera Uria ${ }^{1, *}$, Francisco Martín Romero², Sergey Sedov ${ }^{2}$, Elizabeth Solleiro Rebolledo ${ }^{2}$

${ }^{1}$ Posgrado en Ciencias de la Tierra, Universidad Nacional Autónoma de México. Ciudad Universitaria, Av. Universidad 3000, Coyoacán, 04520, CDMX, México.

${ }^{2}$ Instituto de Geología, Universidad Nacional Autónoma de México. Ciudad Universitaria, Av. Universidad 3000, Coyoacán, 04520, CDMX, México.

*Autor para correspondencia: (M. Y. Rivera Uria)petuzza@gmail.com

\footnotetext{
Cómo citar este artículo:

Rivera Uria, M.Y., Martín Romero, F., Sedov, S., Solleiro Reobolledo, E., 2020, Carbonatos pedogénicos para el tratamiento del drenaje ácido de mina (DAM). Experimentos de laboratorio: Boletín de la Sociedad Geológica Mexicana, 72 (1), A250919. http://dx.doi.org/10.18268/BSGM2020v72nla250919
}

Manuscrito recibido: 28/11/2017 Manuscrito corregido: 11/10/2018 Manuscrito aceptado: 20/11/2018

La revisión por pares es responsabilidad de la Universidad Nacional Autónoma de México.

\section{RESUMEN}

Una de las problemáticas ambientales principales de las zonas mineras es la generación del denominado drenaje ácido de mina (DAM), que tiene un impacto negativo para el ambiente y la salud humana debido a las altas concentraciones de elementos potencialmente tóxicos (EPT). Existen varios tratamientos que utilizan diferentes tipos de rocas para el control de drenaje ácido, entre las cuales la caliza es muy utilizada debido a su excelente potencial de neutralización. Otro material que reúne estas características es el carbonato de origen pedogénico; sin embargo, no hay estudios que valoren su utilidad. Se estudió la efectividad de un edafosedimento carbonatado y una caliza en el tratamiento del DAM de una mina abandonada. Se realizó una caracterización química, mineralógica, geoquímica y micromorfológica de los dos materiales formados de carbonatos de calcio con diferente origen (edafosedimento y caliza.). Se realizaron pruebas en lotes, utilizando edafosedimento carbonatado-DAM con relaciones 1:2.5 y 1:20 (sólido liquido $_{\mathrm{mL}}$ ), y caliza-DAM con las mismas relaciones. Se midió la cinética del $\mathrm{pH}$ y conductividad eléctrica a la primer hora y posteriormente cada 24 hs hasta las 259 hs. Los valores de pH finales en ambos experimentos cambiaron de ácidos a neutros. Por otro lado, la conductividad eléctrica bajó más en los tratamientos con caliza que en el edafosedimento sobre todo en la relación 1:2.5, lo que se atribuye a la presencia de sales. Adicionalmente, los resultados químicos en el DAM después del tratamiento indican una retención del $100 \%$ de $\mathrm{Cu}, \mathrm{Fe}, \mathrm{Ni}, \mathrm{Al}$ y $\mathrm{Cd}$ en la caliza y en el edafosedimento una relación 1:2.5. En cuanto al Zn se logró una retención con valores por debajo de los límites máximos permisibles. Por el contrario, en todos los tratamientos se libera Mn de la fase sólida, y tiende hacia una mayor concentración en el tratamiento con caliza. El edafosedimento carbonatado resultó ser eficiente en la neutralización y precipitación de los EPT y más viable la relación 1:20, ya que la relación 1:2.5 presentó altas concentraciones de $\mathrm{Na}^{+}$y $\mathrm{Cl}^{-}$que pueden ser problema de salinidad.

Palabras clave: DAM, tratamiento pasivo, caliza, carbonatos secundarios, elementos potencialmente tóxicos.

\section{ABSTRACT}

One of the main problems in mining areas is the generation of acid mine drainage (AMD), which has a negative impact on the environment and human health due to the high concentrations of potentially toxic elements. Several treatments exist that use different types of rocks and processes for the control of acid drainage; among them, limestone is an excellent neutralizer. Another neutralizer is carbonate with pedogenic origin; however, there are no studies evaluating its usefulness. The present work aims to evaluate experimentally the capacity of a carbonate pedosediment and a limestone to neutralize and retain the potentially toxic elements (PTE) of acid drainage from an abandoned mine. The study involved a chemical, mineralogical, geochemical, and micromorphological characterization of the two materials formed from calcium carbonates with different origin (pedosediment and limestone), with the aim of conducting batch treatment tests using pedosediment and $A M D$ with ratios of $1: 2.5$ and 1:20 (solid: liquid $_{m L}$ ), limestone and $A M D$ with the same ratios. The $\mathrm{pH}$ and electrical conductivity of the drainage were measured at the first hour and then every 24 hours until stabilization, which occurred at 259 hours with each neutralizer. The final $p H$ values in both experiments changed from acid to neutral. On the other hand, the electrical conductivity was lower in treatments with limestone than in the pedosediment, especially in the ratio of 1:2.5, possibly associated with a higher salt content. Additionally, the chemical results in the AMD after the tests indicates a high retention of $\mathrm{Cu}, \mathrm{Fe}, \mathrm{Ni}, \mathrm{Al}$ and $\mathrm{Cd}$ in the limestone and pedosediment relation 1:2.5. Zn retention was achieved up to concentrations below the maximum permissible limits. In contrast, in all treatments $\mathrm{Mn}$ is released from the solid phase, with a higher concentration in the limestone. The pedosediment is considered efficient in the neutralization and precipitation of the PTE. Results were better with the ratio 1:20, because 1:2.5 had higher concentrations of $\mathrm{Na}^{+}$and Ct that can be a problem of salinity.

Keyrerds: AMD, passive treatment, limestone, secondary carbonates, potentially toxic elements. 


\section{Introducción}

Uno de los problemas que se presenta en las zonas mineras a nivel mundial es la generación de drenaje ácido de mina (DAM), ya que puede producir efectos negativos en los ecosistemas, como la contaminación de cuerpos de agua, suelos y afectación de flora y fauna (Valente y Leal, 2009). Los residuos que provienen de las minas de yacimientos metálicos contienen minerales sulfurados residuales como pirita $\left(\mathrm{FeS}_{2}\right)$, marcasita $\left(\mathrm{FeS}_{2}\right)$ y pirrotita $\left(\mathrm{Fe}_{\mathrm{x}} \mathrm{S}_{\mathrm{x}}\right)$. Estos minerales al estar expuestos al agua y oxígeno generan lixiviados conocidos como drenaje ácido de mina (DAM) (Morin y Hutt, 1997; Udayabhanu y Prasad, 2010).

El DAM tiene la característica de presentar valores de $\mathrm{pH}$ bajos $(<3)$, conductividades eléctricas elevadas $(>800 \mu \mathrm{S} / \mathrm{cm})$, altas concentraciones de sulfatos y metales y metaloides disueltos tales como Pb, Zn, Cu, Cid, Cr, Ni, Co, Al, Sb y As, conocidos como Elementos potencialmente tóxicos ("EPT") (Akcil y Koldas, 2006).

Por las razones antes expuestas se han propuesto diferentes tipos de tratamientos para el control y neutralización del drenaje ácido de mina. En particular, tratamientos pasivos se han desarrollado en los últimos 20 años con el objetivo de contar con tecnologías que involucren procesos físicos, químicos y biológicos, propios de los ambientes naturales, para promover la neutralización y la retención de los EPT (López et al., 2002). Entre los diferentes sistemas de tratamiento están los humedales aerobios, humedales anaerobios, drenajes anóxicos, barreras reactivas permeables, sistemas sucesivos de producción alcalina, fitotecnologías, reactores bioquímicos, entre otros (Taylor et al., 2005; U.S. EPA, 2014).

Uno de los materiales más utilizados, en los sistemas de tratamiento pasivo, es la roca caliza. Diversos estudios comprueban que es un excelente material para la neutralización de los drenajes ácidos y retención de EPT (U.S. EPA, 1998; Romero et al., 2011; Corrales-Pérez y Romero, 2013).

La caliza (conformada de carbonato de calcio, principalmente) reacciona con un medio ácido y empieza a disolverse liberando $\mathrm{Ca}^{2+}$ y bicarbonatos $\left(\mathrm{HCO}_{3}^{-}\right)$. Por lo tanto, el $\mathrm{pH}$ del medio sube hasta llegar a valores neutros y muchos de los metales que se encuentran disponibles como $\mathrm{Cu}^{2+}$, $\mathrm{Zn}^{2+}, \mathrm{Cd}^{2+}, \mathrm{Fe}^{3+}, \mathrm{Al}^{3+}$, pasan a la fase sólida por precipitación y/o sorción, y como consecuencia bajan las concentraciones del medio acuoso.

Sin embargo, varios autores comentan que la "vida útil" de la caliza se puede ver limitada por la precipitación de los Fe-oxi-hidróxidos y de yeso, que forman costras que recubren la caliza y la inactivan, limitando el proceso de neutralización (Santomartino y Webb, 2007; Offeddu et al., 2015). Si bien está documentado el potencial de la caliza y sus limitantes, los denominados carbonatos pedogénicos o carbonatos secundarios que se desarrollan en ambientes terrestres, especialmente en los sistemas edáficos, no han sido estudiados.

Estos carbonatos se forman por dos procesos principales, el primero denominado descarbonatación-carbonatación. La descarbonatación inicia cuando el $\mathrm{CO}_{2}$ disuelto en agua forma ácido carbónico que disuelve los carbonatos primarios (calizas, por ejemplo), produciendo bicarbonatos de calcio, los cuales precipitan posteriormente en forma de carbonatos en el suelo (Baird, 2001; Gisbert e Ibáñez, 2008; Boul et al., 2011). En el segundo proceso los carbonatos se forman por disolución de minerales primarios ricos en $\mathrm{Ca}^{+2}$ como las plagioclasas. Siguiendo un camino similar a la descarbonatación anterior, hay formación de ácido carbónico en el suelo que va disolviendo las plagioclasas liberando un $\mathrm{H}^{+}$y captando $\mathrm{Ca}^{2+}$. Se forman así los bicarbonatos cálcicos y, si las condiciones en el sistema presentan altas tasas de evaporación, como consecuencia precipitan los carbonatos secundarios en los suelos.

La precipitación y acumulación de los carbonatos en los suelos es un proceso pedogenético muy común que se da en los ambientes áridos y semiáridos (Krasilnikov et al., 2013).

En las regiones ubicadas al norte de México, donde la actividad minera se encuentra más desarrollada, no se observan afloramientos de rocas calizas. Sin embargo, los carbonatos secundarios 
son muy abundantes en los suelos y edafosedimentos de estas zonas, como en el caso del estado de Sonora. Estos carbonatos pueden proponerse como una nueva alternativa para los tratamientos de los drenajes ácidos de mina.

En este trabajo se presentan los resultados experimentales de pruebas en lote del uso y efectividad de los carbonatos secundarios de una secuencia edafosedimentaria, como barrera pasiva para la neutralización del drenaje ácido de mina.

Se planteó la hipótesis de que los carbonatos presentes en el edafosedimento tienen la misma capacidad de neutralizar al DAM que la caliza, que actualmente se usa de manera eficiente en la neutralización del DAM de Taxco, Guerrero, por lo que se realizaron pruebas con ambos tipos de carbonatos para comparar su eficiencia en el control del drenaje ácido de minas.

\section{Metodología}

\subsection{MUESTREO Y GARACTERIZAGIÓN DEL EDAFOSEDIMENTO GARBONATADO, GALIZA Y EL DRENAJE ÁCIDO DE MINA}

Se colectaron tres tipos de muestras: 1-materiales edafosedimentarios carbonatados (Figura 1A) ubicados al norte del estado de Sonora (N31 ${ }^{\circ} 02^{\prime} 26.3^{\prime \prime}, \mathrm{W} 112^{\circ} 03^{\prime} 15.2^{\prime \prime}, 548 \mathrm{msnm}$ ), 2- la roca caliza (Figura 1B) y 3-drenaje ácido de mina (Figura 1C) en Taxco, Guerrero (N18 $31^{\prime} 53^{\prime \prime}$ y O99'35'37'", 1412 msnm).

El edafosedimento carbonatado está compuesto por los siguientes horizontes: C-2Bgk 1-2Bgk2-3C4Bk-5Bgk-5BCk-5Ck-6Bk-7Bg. Se colectó $1 \mathrm{~kg}$ de cada horizonte y posteriormente se mezclaron todos los horizontes para obtener una muestra compuesta (CS). Por otro lado, se recolectaron $2 \mathrm{~kg}$ de caliza (CT). Tanto la caliza como el edafosedimento se molieron y tamizaron a malla 200 (diámetro $=0.074 \mathrm{~mm}$ ).

A las dos muestras se les determinó pH, con un equipo Denver Instrument Ultrabasic, y conductividad eléctrica, con equipo Oakaton 700, para lo cual se preparó una mezcla con agua destilada en una relación 1:20 y agitación de 1h. También se realizaron pruebas de potencial de neutralización (PN) conforme al protocolo de prueba de la NOM-141 (Semarnat, 2003), por triplicado.

La composición química se determinó por fluorescencia de rayos $\mathrm{X}$ (FRX) con el equipo Thermo Scientific Niton LX31. Se identificó la mineralogía por medio de la difracción de rayos $\mathrm{X}$ (DRX) con el equipo Shimadzu XRD-6000. Para la identificación de las fases mineralógicas se utilizó el programa High Score Plus, posteriormente se realizó un análisis semicuantitativo (semicuant) por medio del Reference Intensity Ratio (RIR), basado en la relación de altura de pico relativo neto de la línea más fuerte de la fase y de la línea más fuerte del corindón.

Adicionalmente se tomaron muestras inalteradas para la elaboración de láminas delgadas de los horizontes: 2Bgk, 4Bk y 5Bgk del edafosedimento y de la caliza para estudios micromorfológicos. Las muestras se secaron y se impregnaron con resina Poliéster, posteriormente se realizaron cortes hasta obtener una lámina delgada con un grosor de aproximadamente 30 micras. Las láminas fueron observadas y descritas con ayuda de un microscopio petrográfico Olympus BX 51 y una cámara Cool Snap.

Con respecto al drenaje ácido de mina (DAM), se midió en laboratorio su $\mathrm{pH}$ y su conductividad eléctrica (CE). Posteriormente, el DAM se filtró con papel de nitrocelulosa con diámetro de $0.45 \mu \mathrm{m}$ para los análisis químicos. Para la cuantificación de metales y metaloides solubles se utilizó un equipo ICP-OES Perkin Elmer modelo 8300DV. Para la cuantificación de los cationes y aniones mayoritarios se empleó cromatografía iónica con el equipo Metrohm 883 Basic IC Plus.

\subsection{PRUEBA DE NEUTRALIZAGIÓN GARBONATOS- DRENAJE ÁGIDO}

Se realizaron pruebas en lotes en laboratorio donde se utilizaron dos tipos de relaciones (solido: líquido) tanto para la caliza (CT) como para el edafosedimento carbonatado (CS), con una 

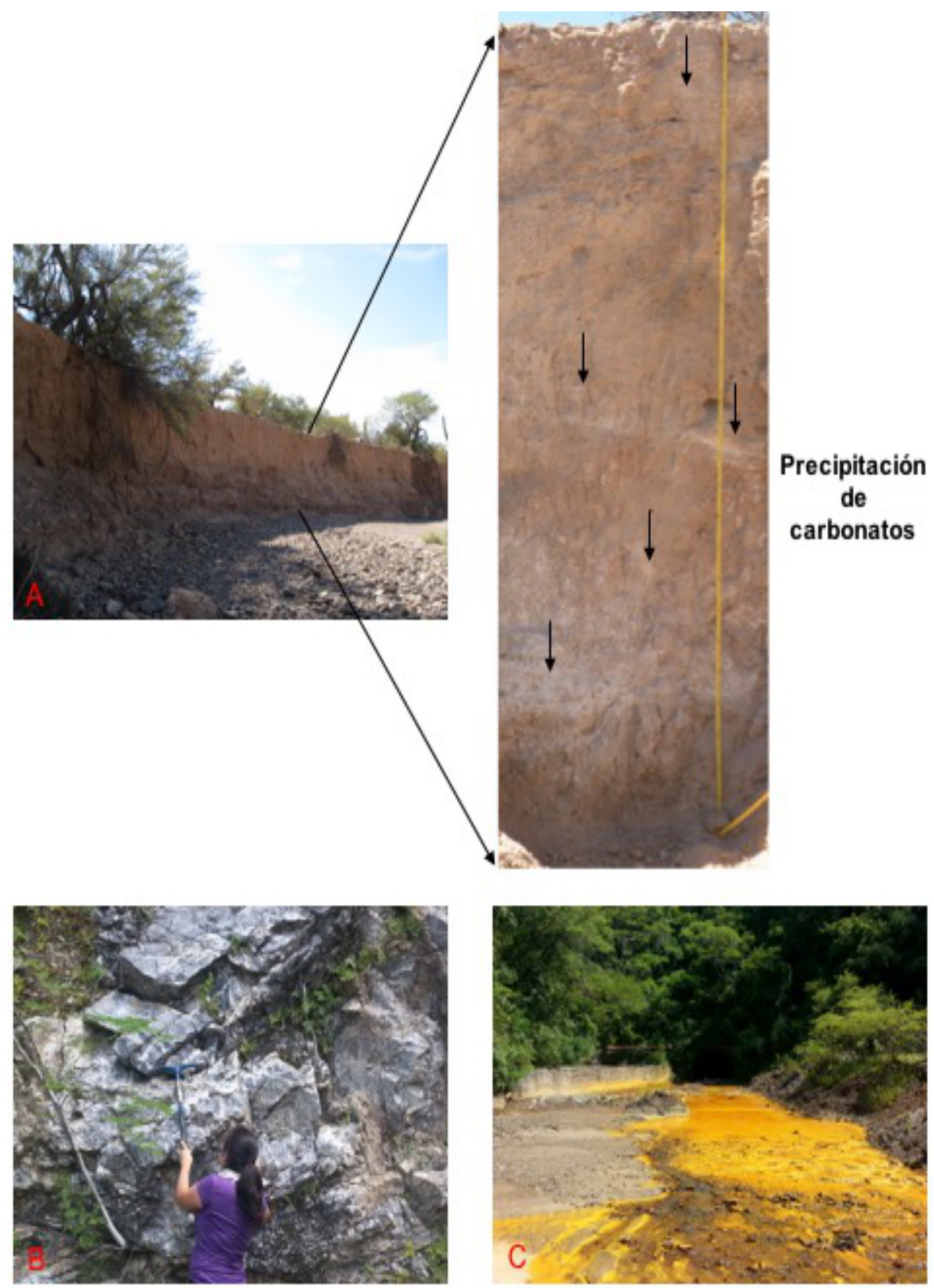

Figura 1 A- El perfil la Cantera, identificando las diferentes profundidades de precipitación de los carbonatos secundarios, en el estado de Sonora. B- Toma de muestra de caliza. C- Drenaje ácido saliendo por el Socavón de la mina subterránea, Taxco, Guerrero. 
relación de 1:20, en la que se pesaron $1.5 \mathrm{~g}$ de sólido y $30 \mathrm{ml}$ de líquido, y una relación 1:2.5, en la que se pesaron $12 \mathrm{~g}$ de sólido y $30 \mathrm{ml}$ de líquido. Se hicieron tres repeticiones para cada prueba. Se midió el pH y la CE cuando se mezclaron las muestras. Posteriormente, todos los tratamientos se agitaron utilizando un agitador automático Thermo Scientific Max 2000 a 200 rpm. Se tomó lectura de los mismos parámetros a la primer hora y a las 18hs, y después cada $24 \mathrm{hs}$ hasta las 259 hs, cuando el $\mathrm{pH}$ y la conductividad eléctrica se estabilizaron.

Finalmente todas las muestras se filtraron con una membrana de nitrocelulosa con diámetro de $0.45 \mu \mathrm{m}$ y se refrigeraron para la medición de las concentraciones solubles de metales y metaloides con ICP-OES Perkin Elmer modelo 8300DV. Los cationes y aniones mayoritarios se midieron por cromatografía iónica, utilizando un equipo Metrohm 883 Basic IC Plus.

Las concentraciones de los EPT de la fase líquida de todos los tratamientos se compararon entre sí y con los límites máximos permisibles de contaminantes en las descargas de aguas residuales en aguas y bienes nacionales (NOM-001SEMARNAT-1996 y la NOM-127-SSA1-1994).

Finalmente las muestras sólidas de ambos tratamientos (CT y CS) fueron analizadas para la identificación de los minerales secundarios que se formaron. Se identificaron por difracción de rayos $\mathrm{X}$, se utilizó un equipo Shimadzu XRD-6000 y un programa High Score Plus. Posteriormente con el mismo programa se realizó un análisis semicuantitativo por medio del RIR (Reference Intensity Ratio).

\section{Resultados y Discusiones}

\subsection{GARAGTERIZAGIÓN DEL EDAFOSEDIMENTO Y GALIZA}

La Tabla 1 muestra la composición química elemental del edafosedimento (CS) y de la caliza (CT), determinados por FRX. Los elementos predominantes en CT son: $\mathrm{Ca}=42.65 \%$,
$\mathrm{Fe}=0.17 \%, \mathrm{Mn}=1292.7 \mathrm{mg} / \mathrm{kg}, \mathrm{Ni}=118.41 \mathrm{mg} /$ kg. En CS los elementos predominantes son: $\mathrm{Ca}=11.9 \%, \mathrm{Fe}=1.4 \%$ y $\mathrm{Mn}=694.6 \mathrm{mg} / \mathrm{kg}$.

Los resultados de $\mathrm{pH}$ y conductividad eléctrica (CE) mostraron que CT presentó un $\mathrm{pH}_{\text {promedio }}=8.2$ y una $\mathrm{CE}_{\text {promedio }}=0.67 \mathrm{mS} / \mathrm{cm}$, y $\mathrm{CS}$ tiene un $\mathrm{pH}_{\text {promedio }}=9.36$ y una $\mathrm{CE}_{\text {promedio }}=1.94 \mathrm{mS} / \mathrm{cm}$. El potencial de neutralización (PN) de CT fue mayor, con una concentración $\mathrm{PN}_{\text {promedio }}=1937.6$ $\mathrm{kg}_{\mathrm{CaCO} 3} /$ Ton, mientras que CS fue más bajo con $\mathrm{PN}_{\text {promedio }}=526.2 \mathrm{~kg}_{\mathrm{CaCO}_{3}} /$ Ton.

En la Tabla 2 se encuentran los resultados de la difracción de rayos X. Se identificó una fase única en CT compuesta de calcita $\left(\mathrm{CaCO}_{3}\right)$, que se conformó con el RIR, que reportó el 100\%. Por el contrario, en CS se identificaron varios minerales: cuarzo $\left(\mathrm{SiO}_{2}\right)$, calcita $\left(\mathrm{CaCO}_{3}\right)$, illita $\left(\mathrm{K}, \mathrm{H}_{3} \mathrm{O}\right)(\mathrm{Al}, \mathrm{Mg}, \mathrm{Fe})_{2}(\mathrm{SiAl})_{4} \mathrm{O}_{10}(\mathrm{OH})_{2}\left(\mathrm{H}_{2} \mathrm{O}\right)$, actinolita $\left(\mathrm{Ca}_{2}\left(\mathrm{Mg}, \mathrm{Fe}^{2+}\right)_{5} \mathrm{Si}_{8} \mathrm{O}_{22}(\mathrm{OH})_{2}\right.$, feldespato sódico $\left(\mathrm{NaAlSi}_{3} \mathrm{O}_{8}\right)$ y moscovita $\left(\mathrm{KAl}_{2}\left(\mathrm{AlSi}_{3} \mathrm{O}_{10}\right)(\mathrm{OH})_{2}\right)$. El resultado semi-cuantitativo RIR mostró una predominancia de cuarzo con $24 \%$, illita $16 \%$, calcita $15 \%$, muscovita $11 \%$, feldespatos sódicos $9 \%$, caolinita $5 \%$ y actinolita $1 \%$.

Por otro lado, en el estudio micromorfológico se observó que CT tiene una textura fina carbonatada dominando el tamaño micrítico, en cuya matriz se observan foraminíferos plantónicos formados por carbonatos biogénicos, como se

Tabla 1. Resultados químicos del edafosedimento carbonatado (CS) y caliza (CT).

\begin{tabular}{|c|c|c|}
\hline Muestra & Caliza & $\begin{array}{c}\text { Edafosedimento } \\
\text { Carbonatado }\end{array}$ \\
\hline pH & 8.2 & 9.3 \\
\hline $\mathrm{CE} \mathrm{mS} / \mathrm{cm}$ & 0.67 & 1.94 \\
\hline $\mathrm{PN} \mathrm{kg} \mathrm{CaCO}_{3} /$ Ton jal & 1038.6 & 535.6 \\
\hline $\mathrm{Fe} \%$ & $1.7 \pm 0.01$ & $1.4 \pm 0.01$ \\
\hline $\mathrm{Ca} \%$ & $42.65 \pm 0.1$ & $11.9 \pm 0.06$ \\
\hline $\mathrm{Pb} \mathrm{mg} / \mathrm{kg}$ & $<\mathrm{LD}$ & $19.5 \pm 2.7$ \\
\hline Zn mg/kg & $44.7 \pm 12.8$ & $46.6 \pm 5.8$ \\
\hline $\mathrm{Cu} \mathrm{mg/kg}$ & $<\mathrm{LD}$ & $59.3 \pm 0.2$ \\
\hline $\mathrm{Ni} \mathbf{m g} / \mathbf{k g}$ & $118.41 \pm 0.3$ & $<\mathrm{LD}$ \\
\hline Mn mg/kg & $1292.7 \pm 102$ & $694.6 \pm 38.9$ \\
\hline $\mathrm{Cr} \mathrm{mg} / \mathrm{kg}$ & $29.7 \pm 2.4$ & $52.1 \pm 9.5$ \\
\hline
\end{tabular}

<LD: por debajo del límite de detección. 
observa en la Figura 2C. También se identificaron fracturas rellenas de carbonatos de tamaño esparítico, que se observan en la Figura 2D.

El material que constituye CS está compuesto por horizontes tipo $\mathrm{Bk}$, que presentan una estructura en bloques subangulares. En la Figura 2A se observa la presencia de los carbonatos secundarios en forma de micrita, los cuales están situados principalmente en los poros, estando algunos de los agregados completamente carbonatados. La Figura 2B muestra zonas enriquecidas con material fino compuesto de arcilla y hierro, lo que le confiere una coloración pardo-rojiza.

La mineralogía está dominada por cuarzo y plagioclasas de tamaño arena, con una baja proporción de ferromagnesianos y un intemperismo intermedio. Se observan, también, fragmentos de suelo redondeados y re-trabajados, asociados a una pedogénesis anterior.

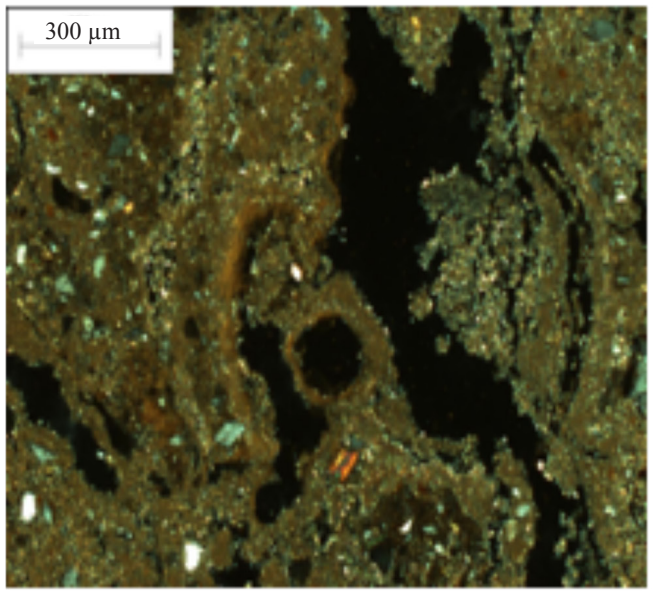

$\mathrm{A}-2 \mathrm{BgK}_{2}$

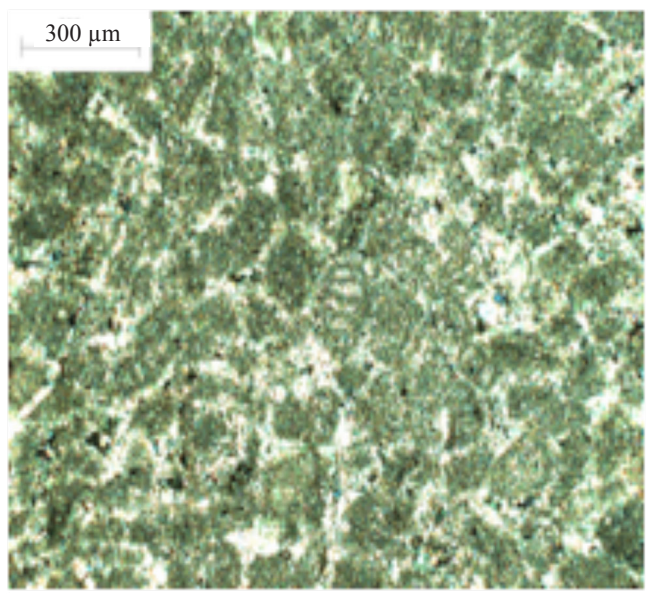

C-caliza, foraminifero bentónico

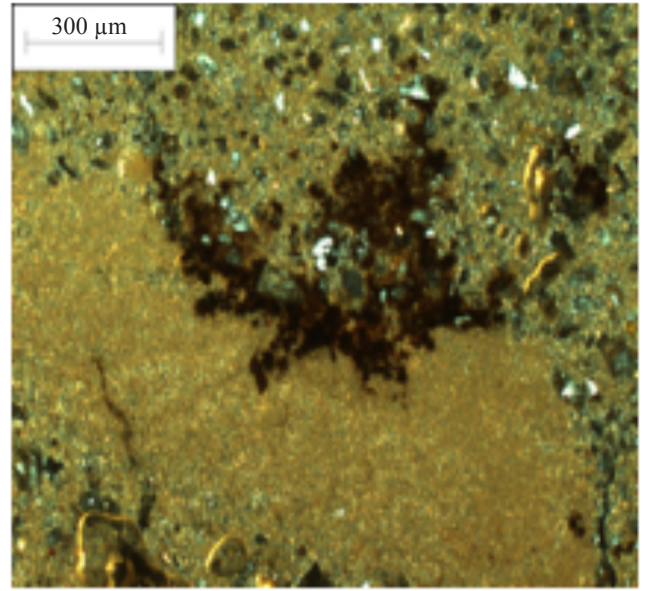

\section{B-5BgK}

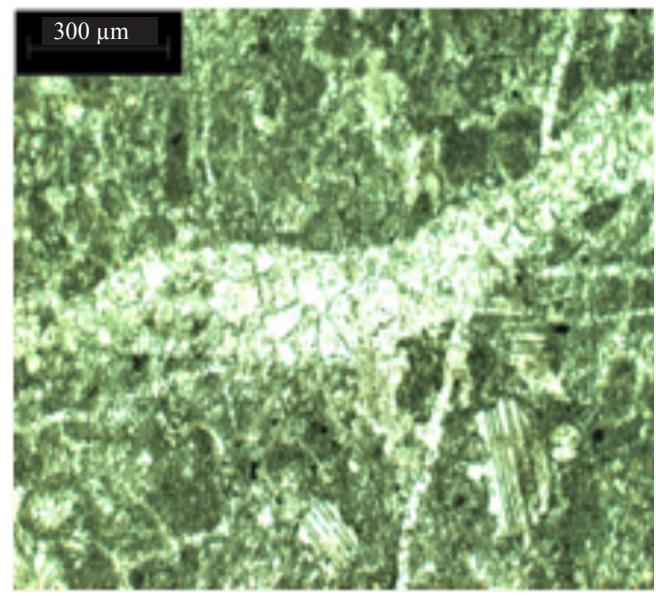

D-matriz carbonatada.

Figura 2 Microfotografías del perfil la Cantera. A- Carbonatos de tamaño micrítico ubicados en poros y matriz de suelo. B- Hidróxidos de Fe en matriz fina carbonatada. La caliza: C- Foraminíferos; D- Matriz completamente carbonatada. 
A través de los estudios químicos y mineralógicos se identificaron y cuantificaron los carbonatos presentes en los dos tipos de muestras. Se demostró que la caliza tiene el doble de potencial de neutralización y con el estudio semicuantitativo RIR se corroboró que la muestra está conformada al 100\% de calcita, además de reconocer que el origen de estos carbonatos está asociado a los ambientes marinos.

En el edafosedimento se identificó que, además de carbonatos, éste presenta varios tipos de minerales en mayor predominancia, como el cuarzo, todo ello basado en el resultado del semicuantitativo RIR, que cuantificó que solo tiene el 15\% de calcita. El potencial de neutralización indicó que el edafosedimento tiene menor contenido de carbonatos, a lo que se interpreta que predominan otro tipo de minerales. El origen de estos carbonatos está asociado a un ambiente terrestre, específicamente ambientes semi-áridos donde hay una mayor evaporación en el medio para la precipitación de los carbonatos.

\subsection{GARAGTERIZAGIÓN QUÍMICA DEL DRENAJE ÁGIDO DE MINA (DAM)}

Los resultados se encuentran en la Tabla 3, que muestra la caracterización realizada al DAM antes de las pruebas. El color del líquido es anaranjado-rojizo, atribuido a la oxidación del Fe, el pH es ácido $\mathrm{pH}=2.3$, y la conductividad eléctrica $\mathrm{CE}=6$ $\mathrm{mS} / \mathrm{cm}$, la cual se considera elevada. Los cationes mayoritarios predominantes son $\mathrm{Mg}^{2+}=297.7$ $\mathrm{mg} / \mathrm{L}$ y $\mathrm{Ca}^{2+}=269.3 \mathrm{mg} / \mathrm{L}$, y en menor concentración $\mathrm{Na}^{+}=14.5 \mathrm{mg} / \mathrm{L}$ y K $\mathrm{K}^{+}=93.2 \mathrm{mg} / \mathrm{L}$. Los aniones determinados fueron $\mathrm{SO}_{4}{ }^{2-}=5503.1$ $\mathrm{mg} / \mathrm{L}, \mathrm{Cl}^{-}=12.7 \mathrm{mg} / \mathrm{L}, \mathrm{F}=9.9 \mathrm{mg} / \mathrm{L}_{\text {y NO}} \mathrm{NO}^{3-}=4.3$ $\mathrm{mg} / \mathrm{L}$.

Los elementos potencialmente tóxicos que se identificaron en orden descendiente

Tabla 2. Difracción de rayos $X$ y análisis semicuantitativo (RIR) de caliza, edafosedimento, CS 1:20 y CT 1:20.

\begin{tabular}{|c|c|c|c|}
\hline Muestra & Fases Identificadas & Fichas & $\begin{array}{c}\text { Semicuant (RIR) } \\
\%\end{array}$ \\
\hline Caliza & Calcita & ICSD 98 004 0107 & 100 \\
\hline & Cuarzo & ICSD 98 008 3849 & 24 \\
\hline & Caolinita & ICSD 98 0031135 & 5 \\
\hline \multirow{2}{*}{ Edafosedimento carbonatado } & Illita & ICSD 98 016 6962 & 16 \\
\hline & Calcita & ICSD 98 002 8827 & 15 \\
\hline & Actinolita & ICSD 98 002 4900 & 1 \\
\hline \multirow{2}{*}{ Tratamiento CT 1:20 } & Feldespato Na & ICSD 98 008 8899 & 9 \\
\hline & Muscovita & ICSD 98 018 0081 & 11 \\
\hline & Calcita & ICSD 98 004 0107 & 86 \\
\hline \multirow{2}{*}{ Tratamiento CS 1:20 } & Yeso & ICSD 98 002 7875 & 14 \\
\hline & Cuarzo & ICSD 98 008 3849 & 18 \\
\hline & Caolinita & ICSD 98 0031135 & 3 \\
\hline & Illita & ICSD 98 016 6962 & 15 \\
\hline & Calcita & ICSD 98 002 8827 & 9 \\
\hline & Actinolita & ICSD 98 002 4900 & 2 \\
\hline & Yeso & ICSD 98 002 7221 & 35 \\
\hline
\end{tabular}


de concentración fueron: $\mathrm{Zn}=243.55 \mathrm{mg} / \mathrm{L}$, $\mathrm{Fe}=221.94 \mathrm{mg} / \mathrm{L}, \quad \mathrm{Al}=65.24 \mathrm{mg} / \mathrm{L}, \quad \mathrm{Si}=15.57$ $\mathrm{mg} / \mathrm{L}, \quad \mathrm{Cu}=10.21 \mathrm{mg} / \mathrm{L}, \quad \mathrm{Cd}=3.048 \mathrm{mg} / \mathrm{L}$, $\mathrm{Sr}=0.76 \mathrm{mg} / \mathrm{L}, \mathrm{Ni}=0.22 \mathrm{mg} / \mathrm{L}$. El único elemento que se encontró por debajo de los límites de detección fue el Mn.

Los resultados concuerdan con las características químicas que determinan a un drenaje ácido de mina: $\mathrm{pH}$ acido, $\mathrm{CE}$ elevada, altas concentraciones de elementos potencialmente tóxicos y sulfatos. Estos resultados se compararon con cinco tipos de DAM de México que fueron estudiados por Romero et al. (2008), encontrado que este drenaje ácido (que proviene de Taxco) tiene la mayor concentración de sulfatos y de acidez.

\subsection{PRUEBAS EN LOTE PARA EL TRATAMIENTO DEL DAM}

Los resultados de la cinética del $\mathrm{pH}$ en las pruebas en lote se observan en la Figura 3A y 3B. La neutralización del DAM con CT relación 1:2.5 incrementó el $\mathrm{pH}$ a la primera hora de contacto, con un valor $\mathrm{pH}_{\text {promedio }}=6.0$; a las $18 \mathrm{hs}$ no tuvo un cambio significativo, con $\mathrm{pH}_{\text {promedio }}=6.2$.
Sin embargo, a las 65 hs el pH incrementó considerablemente, con un valor $\mathrm{pH}_{\text {promedio }}=7.0$. A partir de este tiempo de agitación el aumento fue paulatino hasta obtener un valor final de $\mathrm{pH}_{\text {promedio }}=7.3$. En el caso de CS, el $\mathrm{pH}$ a la primera hora de contacto fue de $\mathrm{pH}_{\text {promedio }}=6.4$. A las $18 \mathrm{hs}$ no se registró un cambio significativo, con $\mathrm{pH}_{\text {promedio }}=6.7$. No obstante, a las $41 \mathrm{hs}$ el valor registrado fue de $\mathrm{pH}_{\text {promedio }}=7.3$. Posterior a este tiempo inició la estabilización de este parámetro, que obtuvo un valor final de $\mathrm{pH}_{\text {promedio }}=7.6$.

En la neutralización del DAM con CT relación 1:20 se observó un incremento considerable del pH a la primera hora de agitación, con $\mathrm{pH}_{\text {promedio }}=5.8$. A las 18 hs se registró un valor de $\mathrm{pH}_{\text {promedio }}^{\text {promedio }}=6.4$. A las $41 \mathrm{hs}$ no hubo cambio significativo, con valor de $\mathrm{pH}_{\text {promedio }}=6.8$. Después de este tiempo, el $\mathrm{pH}$ inició la estabilización, con un valor final de $\mathrm{pH}_{\text {promedio }}=7.5$. El tratamiento CS tuvo valores semejantes a CT. A la primera hora de agitación el $\mathrm{pH}$ subió a $\mathrm{pH}_{\text {promedio }}=5.9$. Posteriormente, a las 18hs no se observó un aumento del parámetro; pero a las $41 \mathrm{hs} \mathrm{el} \mathrm{pH}_{\text {promedio }}=6.9$. A partir de esta hora, el incremento fue paulatino hasta alcanzar un valor final de $\mathrm{pH}_{\text {promedio }}=7.5$.

Tabla 3. Resultados químicos de aniones, cationes y elementos potencialmente tóxicos del drenaje ácido de mina (DAM) y de las pruebas de neutralización con caliza (CT) y edafosedimento carbonatado (CS).

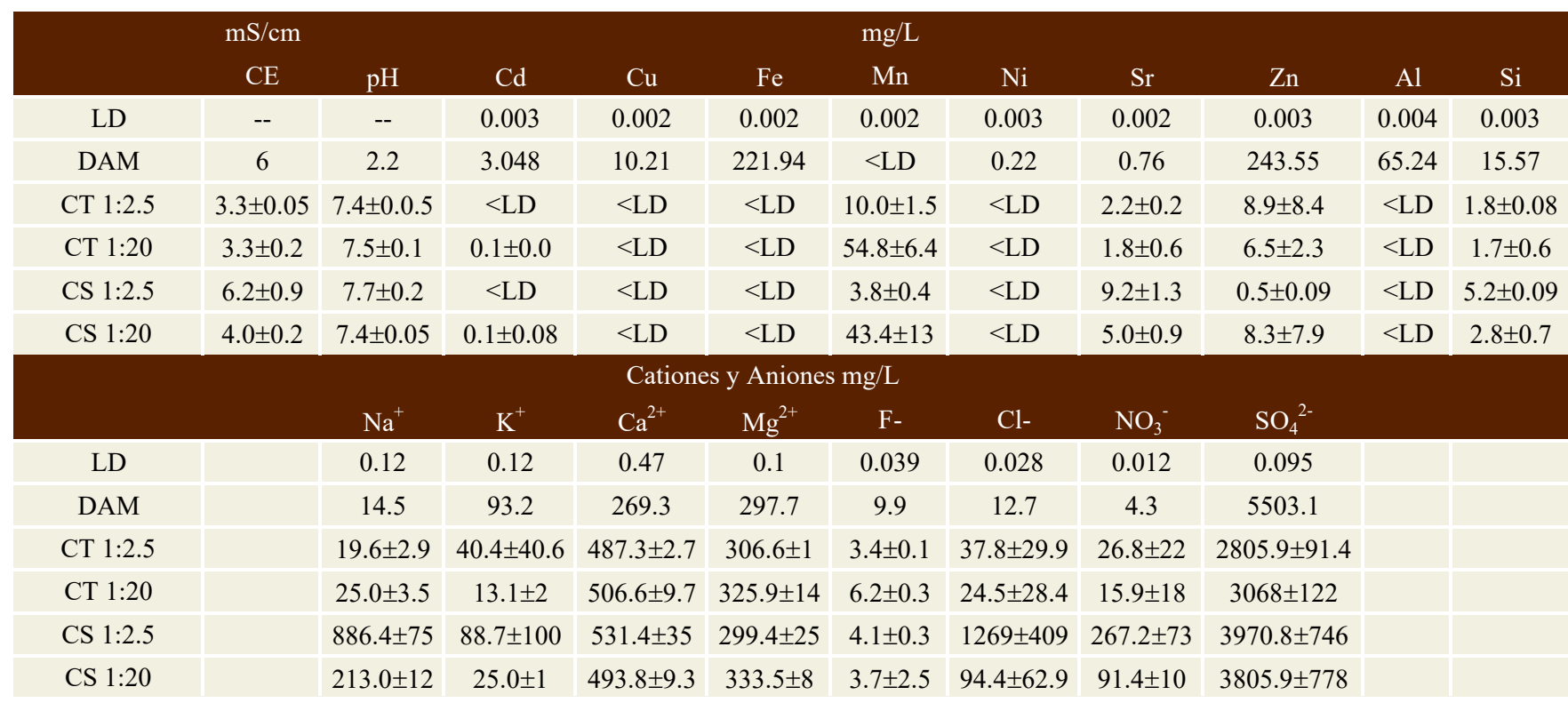

Nota: CT (caliza), CS (edafosedimento carbonatado), <LD (por debajo del límite de detección), LD (límite de detección). 
El contacto entre los dos tipos de sedimentos y el DAM promovió la disolución de la calcita presente y el incremento de la alcalinidad registrado con el monitoreo del $\mathrm{pH}$. Este cambio produjo la liberación de $\mathrm{Ca}^{2+}$ y $\mathrm{H}_{2} \mathrm{CO}_{3}^{-}$, según lo propuesto por Cravotta y Kay (1999) en las siguientes ecuaciones:

$$
\mathrm{CaCO}_{3}(\mathrm{~s})+2 \mathrm{H}^{+} \rightarrow \mathrm{Ca}^{2+}+\mathrm{H}_{2} \mathrm{CO}_{3}^{-}
$$

$$
\mathrm{CaCO}_{3}(\mathrm{~s})+\mathrm{H}_{2} \mathrm{CO}_{3} \rightarrow \mathrm{Ca}^{2+}+2 \mathrm{HCO}_{3}^{-} \text {(2) }
$$

Los protones $\left(\mathrm{H}^{+}\right)$que dan la acidez del DAM son consumidos por los carbonatos formando ácido carbónico $\left(\mathrm{H}_{2} \mathrm{CO}_{3}^{-}\right)$lo que favoreció el incremento del $\mathrm{pH}$. Como los valores finales de pH tanto de CT como de CS en ambas relaciones fueron por arriba de 7 se considera que continuó la disolución de los carbonatos hasta alcanzar

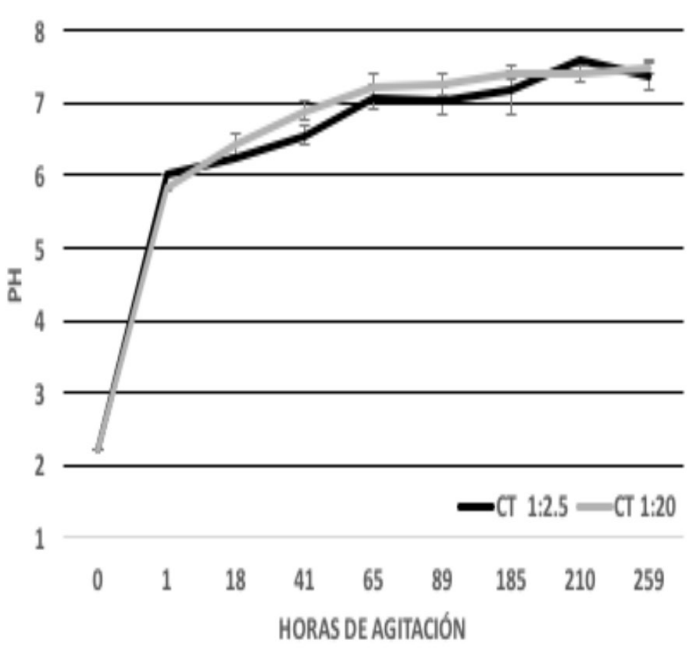

A

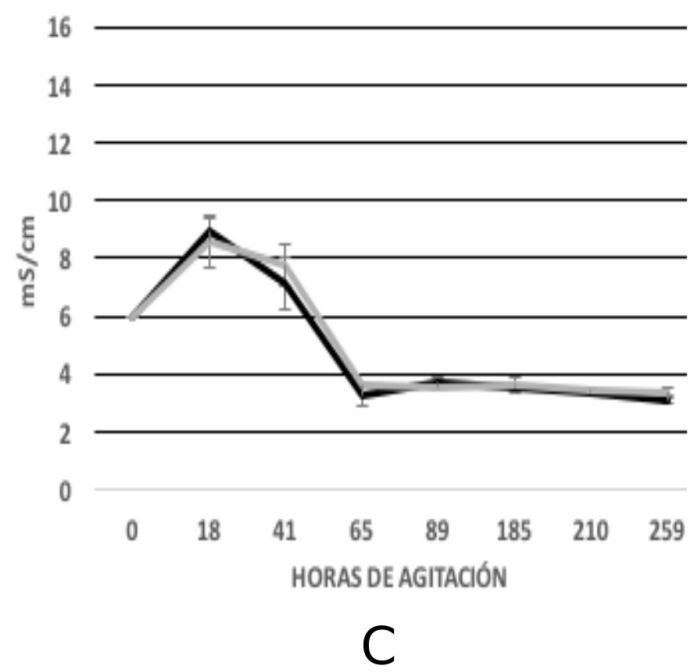

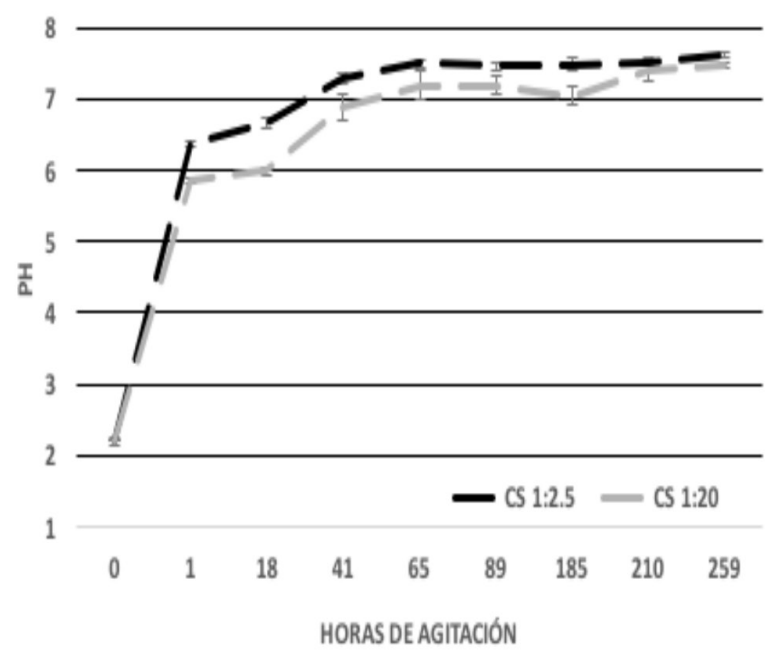

B

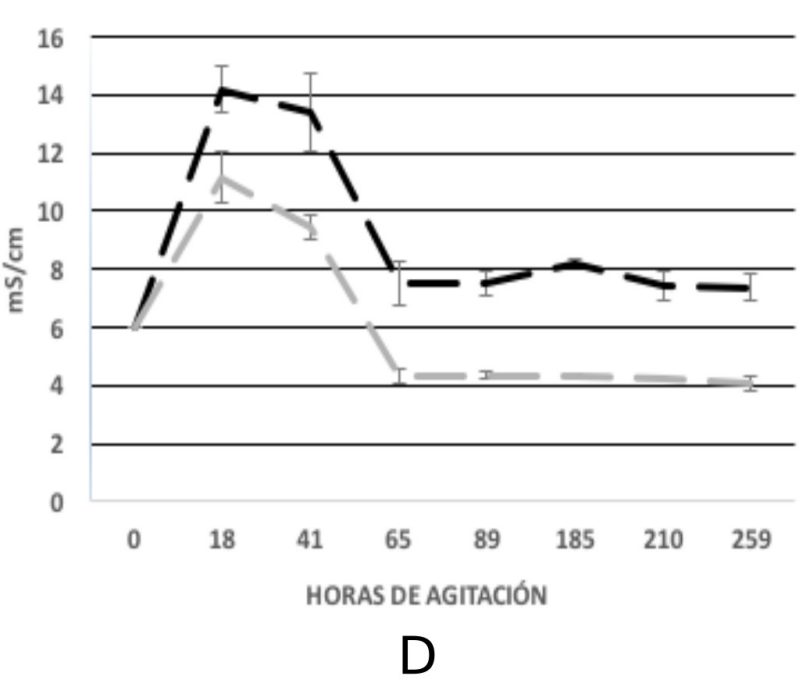

Figura 3 Gráficas que representan el cambio de $\mathrm{pH}$ y conductividad eléctrica durante el tiempo de agitación de los diferentes tratamientos. A- pH del edafosedimento carbonatado. B- Conductividad eléctrica del edafosedimento carbonatado. C- $\mathrm{pH}$ de la caliza. D- Conductividad eléctrica de la caliza. 
la neutralización total. Asta-Andrés et al. (2011) comenta que en el proceso de neutralización del DAM utilizando carbonatos, obtener un $\mathrm{pH}$ final de alrededor de 7 como se observa en la Figura 4A es considerado una neutralización efectiva, ya que para que esto ocurra se debió producir la reacción 2, liberando $\mathrm{Ca}^{2+}$ y bicarbonatos $\left(2 \mathrm{HCO}_{3}{ }^{-}\right)$.

Los resultados de las cinéticas de la conductividad eléctrica se encuentran en la Figura 3C y 3D. En la relación 1:2.5. en CT a las 18 hs de agitación registró un aumento de este parámetro respecto al valor inicial del DAM de $\mathrm{CE}_{\text {promedio }}=8.9 \mathrm{mS} / \mathrm{cm}$. La concentración decreció considerablemente a las $65 \mathrm{hs}$, con $\mathrm{CE}_{\text {promedio }}=3.29 \mathrm{mS} / \mathrm{cm}$. Posterior a este tiempo, inició la estabilización, y alcanzó un valor final de $\mathrm{CE}_{\text {promedio }}=3.09 \mathrm{mS} / \mathrm{cm}$.

Por el contario, CS obtuvo el valor más alto a las $18 \mathrm{hs}$ con $\mathrm{CE}_{\text {promedio }}=14.19 \mathrm{mS} / \mathrm{cm}$, y decreció muy poco a las $41 \mathrm{hs} \mathrm{a} \mathrm{CE}_{\text {promedio }}=13.39 \mathrm{mS} / \mathrm{cm}$. A partir de las 65hs se inició la estabilización de la conductividad, con un valor final de $\mathrm{CE}_{\text {promedio }}=7.3$ $\mathrm{mS} / \mathrm{cm}$.

En la relación 1:20 del tratamiento CT a las 18hs la conductividad eléctrica dio un valor de $\mathrm{CE}_{\text {promedio }}=8.5 \mathrm{mS} / \mathrm{cm}$. Este parámetro cambió a las $41 \mathrm{hs}$, con $\mathrm{CE}_{\text {promedio }}=7.7 \mathrm{mS} / \mathrm{cm}$. A las 65hs la conductividad bajo considerablemente e inició la estabilización, y obtuvo un valor final de $\mathrm{CE}_{\text {promedio }}=3.3 \mathrm{mS} / \mathrm{cm}$. En CS la conductividad eléctrica aumentó a las $18 \mathrm{hs}$ con $\mathrm{CE}_{\text {promedio }}=11.15$ $\mathrm{mS} / \mathrm{cm}$, siendo mayor que en CT. A las $41 \mathrm{hs}$ disminuyó, con $\mathrm{CE}_{\text {promedio }}=9.43 \mathrm{mS} / \mathrm{cm}$. De igual manera que en CT, a las 65 hs empezó la estabilización del parámetro,y su valor final fue de $\mathrm{CE}_{\text {promedio }}=4.05 \mathrm{mS} / \mathrm{cm}$.

Las 18 hs fue la única hora en la que en todos los tratamientos se elevó la conductividad eléctrica, esto se atribuye a la liberación de $\mathrm{Ca}^{2+}$ a la fase acuosa que, junto con los sulfatos y protones se convierten en iones en solución con mayor capacidad de conducir la electricidad. Conforme se va neutralizando el DAM se consume el $\mathrm{Ca}^{2+}$ y los sulfatos y la conductividad decrece excepto en CS relación 1:2.5. Durante las pruebas la conductividad siempre se mantuvo elevada en este tratamiento, que finalmente tuvo un valor mayor respecto al DAM (Figura 4B). Este comportamiento se atribuye a la presencia de sales, lo que se discutirá más adelante.

Los resultados de los aniones y cationes mayoritarios se encuentran en la Tabla 3. En la relación 1:2.5 los aniones más indicativos fueron los sulfatos $\left(\mathrm{SO}_{4}{ }^{2-}\right)$ y los cloruros $\left(\mathrm{Cl}^{-}\right)$. La concentración de $\mathrm{SO}_{4}{ }^{2-}$ bajó al final de las pruebas en lote en los dos tipos de materiales, siendo más efectivo en CT con $\mathrm{SO}_{4}^{2-}$ promedio $=2805.9 \mathrm{mg} / \mathrm{L}$, seguido de CS con $\mathrm{SO}_{4}{ }^{2-}$ promedio $=3970.8 \mathrm{mg} / \mathrm{L}$. Por el contrario, los $\mathrm{Cl}^{-}$mostraron una línea diferente. Aumentó la concentración final de CS con $\mathrm{Cl}^{-}{ }_{\text {promedio }}=268.8 \mathrm{mg} / \mathrm{L}$ (1:2.5), y CT presentó un valor final $\mathrm{Cl}^{-}$promedio $=37.8 \mathrm{mg} / \mathrm{L}$.

En cuanto a los cationes mayoritarios el calcio $\left(\mathrm{Ca}^{2+}\right)$ y el sodio $\left(\mathrm{Na}^{+}\right)$presentaron resultados más significativos. El $\mathrm{Ca}^{2+}$ aumentó su concentración final de las pruebas en lote respecto al DAM, donde CT tuvo una concentración de $\mathrm{Ca}^{2+}{ }_{\text {promedio }}=487.3$ $\mathrm{mg} / \mathrm{L} \mathrm{y} \mathrm{CS} \mathrm{de} \mathrm{Ca}^{2+}{ }_{\text {promedio }}=531.4 \mathrm{mg} / \mathrm{L}$. En cuanto al $\mathrm{Na}^{+}$, CT no mantuvo cambios significativos al final con $\mathrm{Na}^{+}{ }_{\text {promedio }}=19.6 \mathrm{mg} / \mathrm{L}$. Por el contrario, este catión incrementó en $\mathrm{CS}$ con $\mathrm{Na}_{\text {promedio }}^{+}=$ $886.4 \mathrm{mg} / \mathrm{L}$.

Los resultados finales de los aniones mayoritarios de la relación 1:20 indicaron que los $\mathrm{SO}_{4}{ }^{2-}$ bajaron en CT, con $\mathrm{SO}_{4}{ }^{2-}{ }_{\text {promedio }}=3068.1 \mathrm{mg} / \mathrm{L}$. De igual forma, en CS el valor fue de $\mathrm{SO}_{4}{ }^{2-}$ promedio $=3805.9$ $\mathrm{mg} / \mathrm{L}$. Por otro lado, los $\mathrm{Cl}^{-}$mostraron diferencias. Este anión incrementó ligeramente en CT con $\mathrm{Cl}^{-}{ }_{\text {promedio }}=24.5 \mathrm{mg} / \mathrm{L}$, siendo mayor en CS con $\mathrm{Cl}_{\text {promedio }}^{-}=94.4 \mathrm{mg} / \mathrm{L}$.

De los cationes mayoritarios analizados, el calcio $\left(\mathrm{Ca}^{2+}\right)$ y el sodio $\left(\mathrm{Na}^{+}\right)$fueron los elementos que mostraron cambios considerables al final de las pruebas. $\mathrm{El} \mathrm{Ca}^{2+}$ aumentó su concentración final respecto a la que presentaba el DAM, en CT presentó $\mathrm{Ca}^{2+}{ }_{\text {promedio }}=506.6 \mathrm{mg} / \mathrm{L}$, y lo mismo en $\mathrm{CS}$, con $\mathrm{Ca}^{2+}{ }_{\text {promedio }}=493.8 \mathrm{mg} / \mathrm{L}$. El Na${ }^{+}$se incrementó considerablemente solo en CS con $\mathrm{Na}_{\text {promedio }}^{+}=213 \mathrm{mg} / \mathrm{L}$, mientras que en CT no tuvo un incremento significativo, presentado un valor de $\mathrm{Na}^{+}$promedio $=25 \mathrm{mg} / \mathrm{L}$. 
El $\mathrm{Na}^{+}$y $\mathrm{Cl}^{-}$en los dos tipos de sedimentos mostraron diferente tendencia. Las concentraciones finales de las pruebas en lote fueron altas en CS relación 1:2.5. El incremento final del catión y anión, junto con una conductividad eléctrica elevada, indica la presencia de sales en forma de $\mathrm{NaCl}$ (véase Figura 4C). CS es un edafosedimento que en su caracterización química inicial presentó un $\mathrm{pH}$ muy básico y una alta conductividad eléctrica; estas dos propiedades químicas se atribuyen a la presencia de sales en los suelos. Las sales presentes en CS al entrar en contacto con el DAM se solubilizaron y como consecuencia la conductividad eléctrica no bajó en las pruebas.

Adicionalmente, la presencia de $\mathrm{Na}^{+}$en la solución al final de las pruebas fue considerada
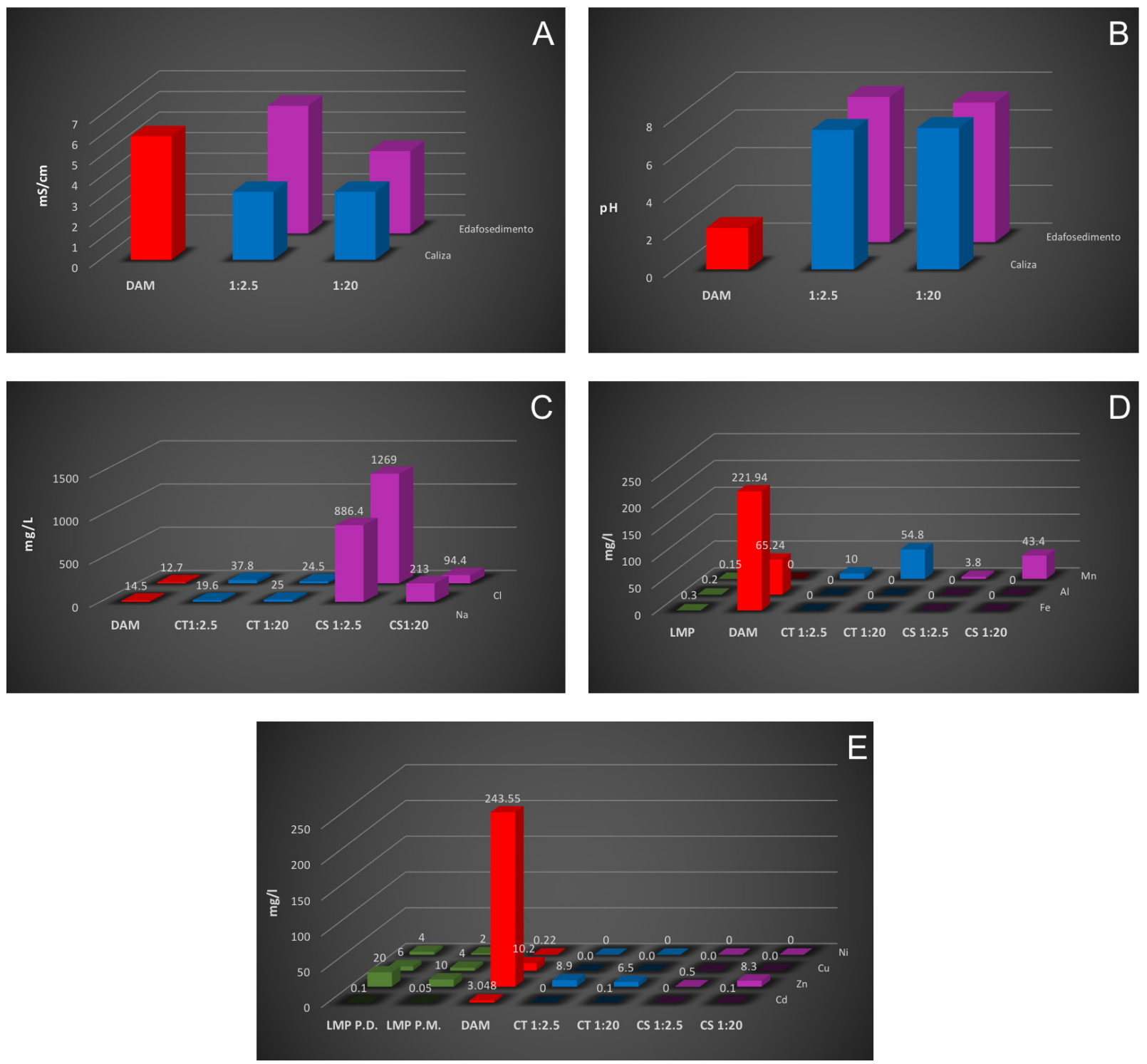

Figura 4 Resultados de la caracterización química. A- pH del drenaje ácido de mina (DAM), edafosedimento y caliza. B- Conductividad eléctrica de drenaje ácido de mina (DAM), edafosedimento y caliza. C- Comparación de los valores iniciales de sodio y cloruros del DAM con los resultados de las pruebas en lote de CS y CT. D- Comparación de los valores iniciales de cadmio, manganeso y zinc del DAM con los resultados de las pruebas en lote de CS y CT. E- Comparativa de los elementos potencialmente tóxicos y los límites máximos permisibles. 
abundante en CS relación 1:2.5, por lo que fue necesario calcular el índice RAS (Relación de Adsorción de Sodio). Este índice calcula la concentración relativa en las aguas de $\mathrm{Na}^{+}$respecto a $\mathrm{Ca}^{2+}$ y $\mathrm{Mg}^{2+}$ e indica la probabilidad de que las aguas, al ser utilizadas para regar en los suelos, formen sales. Se obtuvo que el líquido tratado (1:2.5) presenta un $\mathrm{RAS}=8.13$; se considera que el uso posible del líquido tiene una alta probabilidad de formar sales en contacto con el suelo. Por el contrario, la relación 1:20 presenta un $\mathrm{RAS}=1.8$ valor que indica baja probabilidad de formar sales.

Por otro lado, el aumento del $\mathrm{Ca}^{2+}$ está vinculado a la disolución de los carbonatos de CT y CS en ambas relaciones. Los productos de las reacciones 1 y 2 indican que al disolverse el carbonato se libera $\mathrm{Ca}^{2+}$ hacia la fase liquida, esto concuerda con los resultados finales, donde las concentraciones del $\mathrm{Ca}^{2+}$ al final fueron mayores.

Por el contrario, la concentración de $\mathrm{SO}_{4}{ }^{2-}$ disminuyó en los dos tipos de sedimentos. Esto se explica con la formación de nuevas fases sólidas y el proceso de precipitación dentro de la neutralización del DAM, donde muchos de los cationes $\mathrm{y}$ aniones forman nuevos minerales. La constante disolución del carbonato de calcio dentro de un ambiente ácido promueve la liberación de $\mathrm{Ca}^{2+}$ que, junto con las altas concentraciones de $\mathrm{SO}_{4}{ }^{2-}$ del DAM original, saturan el medio y se produce la precipitación del yeso $\left(\mathrm{CaSO}_{4} \cdot 2 \mathrm{H}_{2} \mathrm{O}\right)$ como se muestra en la ecuación 3 :

$$
\mathrm{Ca}^{2+}+\mathrm{SO}_{4}{ }^{2-}+2 \mathrm{H}_{2} \mathrm{O} \rightarrow \mathrm{CaSO}_{4} \cdot 2 \mathrm{H}_{2} \mathrm{O}_{(\mathrm{s})}
$$

Los resultados del análisis de difracción de rayos $\mathrm{X}$ que se encuentran en la Tabla 2 corrobora la identificación del yeso en los dos tipos de sedimentos, mineral que originalmente no se encuentra en CT y CS. Santomartino y Webb (2007) describen que durante la neutralización del DAM varios tipos de minerales, como el caso del yeso, pueden precipitar cuando hay alta concentración de $\mathrm{SO}_{4}{ }^{2-}$ $(>2000 \mathrm{mg} / \mathrm{L})$. El resultado semicuantitativo (RIR) indica que en CT el yeso tiene un 14\% y para CS un $35 \%$. De igual forma, en ambos tratamientos la calcita todavía se identifica pero en un porcentaje bajo, ello interpretado como una pérdida por su disolución.

El análisis RIR (Reference Intensity Ratio) es un análisis que se basa en la relación del pico más alto de la fase identificada y el pico del corindón; ambos se comparan con la intensidad de los picos en cada fase identificada. Se considera un análisis semicuantitativo que presenta cálculos porcentuales. Los resultados mostraron que CS tuvo mayor porcentaje de formación de yeso que CT, interpretado por la alta intensidad en el pico del yeso. A pesar de que es un cálculo porcentual, se puede interpretar indirectamente que CS tuvo una capacidad mayor de interacción con el DAM. Finalmente, la difracción de rayos X no mostró claramente la formación de otros minerales, pero se sabe que muchos cationes pueden a precipitar en forma de óxidos e hidróxidos de baja cristalinidad (Offeddu et al., 2015) que son difíciles de identificar con este método.

En cuanto a los resultados químicos de los elementos potencialmente tóxicos (EPT) que se muestran en la Tabla 3, estos indican que en la relación 1:2.5, tanto CT como CS, elementos como cadmio $(\mathrm{Cd})$, cobre $(\mathrm{Cu})$, hierro $(\mathrm{Fe})$, niquel (Ni) y aluminio $(\mathrm{Al})$ precipitaron al 100\%. En cambio el zinc $(\mathrm{Zn})$ precipitó parcialmente; en CT presentó un valor de $\mathrm{Zn}_{\text {promedio }}=8.9 \mathrm{mg} / \mathrm{L}$ y en CS $\mathrm{Zn}_{\text {promedio }}=0.5 \mathrm{mg} / \mathrm{L}$. El comportamiento del manganeso $(\mathrm{Mn})$ fue diferente, liberándose hacia la fase líquida con un valor final en CT de $\mathrm{Mn}_{\text {promedio }}=10.0 \mathrm{mg} / \mathrm{L}$ y en CS con $\mathrm{Mn}_{\text {promedio }}=3.8$ $\mathrm{mg} / \mathrm{L}$.

En los resultados de EPT en la relación 1:20 se observa que tanto en CT como en CS los siguientes elementos: $\mathrm{Cu}, \mathrm{Fe}, \mathrm{Ni}$ y $\mathrm{Al}$ precipitaron al 100\%. Solo el Cd presentó una baja concentración, $\mathrm{Cd}_{\text {promedio }}=0.1 \mathrm{mg} / \mathrm{L}$ en ambos sedimentos. Otro elemento que todavía se encontró en la fase líquida fue el $\mathrm{Zn}$, con una concentración en CT de $\mathrm{Zn}_{\text {promedio }}=6.5 \mathrm{mg} / \mathrm{L} \mathrm{y}$ en CS un valor de $\mathrm{Zn}_{\text {promedio }}=8.3 \mathrm{mg} / \mathrm{L}$. El caso del manganeso $(\mathrm{Mn})$ fue similar al de la relación pasada; se liberó durante las pruebas en lote, en 
CT presentó un valor de $\mathrm{Mn}_{\text {promedio }}=54.75 \mathrm{mg} / \mathrm{L} \mathrm{y}$ en CS Mn $n_{\text {promedio }}=43.43 \mathrm{mg} / \mathrm{L}$.

$\mathrm{El}$ incremento del $\mathrm{pH}$ fue un parámetro importante en los procesos de neutralizaciónprecipitación de los EPT. La disolución de los carbonatos durante la interacción con el DAM promovió el incremento del $\mathrm{pH}$. Al darse este incremento, se propició la precipitación de la mayoría de los elementos disueltos. Los resultados indican que durante el tratamiento del DAM con CS y CT el fenómeno geoquímico que controló la movilidad de metales como $\mathrm{Fe}, \mathrm{Al}, \mathrm{Cu}, \mathrm{Ni}$ y Cd y Zn fue la precipitación, aunque también hubo otros procesos como las condiciones redox y los procesos de sorción que intervinieron en su control.

Ha sido reportado que la precipitación de los metales trivalentes, como $\mathrm{Fe}^{3+}$ y $\mathrm{Al}^{3+}$, se da a partir de pH igual a 3 (Hammarstrom et al., 2003; Kalin et al., 2006), lo mismo que el metal divalente con $\mathrm{Cu}^{2+}$ precipita a partir de $\mathrm{pH} 5$, lo que los convirtió en los primeros cationes removidos al $100 \%$ del DAM, al reaccionar a la primera hora de contacto con los dos tipos de carbonatos. Otros metales divalentes, como $\mathrm{Ni}^{2+}$ y $\mathrm{Cd}^{2+}$, precipitaron a un pH cercano a 8 (Tack et al., 1996); estas condiciones se alcanzaron primero en CS a las $65 \mathrm{hs}$ (1:2.5) y 210 hs (1:20), posteriormente en CT a las $185 \mathrm{hs}(1: 20)$ y a las $210 \mathrm{hs}$ (1:2.5); al final de las pruebas se obtuvo la precipitación del 100\% de estos elementos.

Por otro lado, el $\mathrm{Zn}^{2+}$ fue el único elemento que precipitó entre un 90-95\% en todos los tratamientos. Para una eficiencia total de remoción de $\mathrm{Zn}$ se necesitan valores de $\mathrm{pH}$ por arriba de 9 (Kalin et al., 2006), lo que explica que en estas pruebas el $\mathrm{Zn}^{2+}$ todavía se encontró en forma disuelta.

En cuanto al $\mathrm{Mn}^{2+}$, éste se liberó de la fase sólida tanto en CS como en CT. La fluorescencia de rayos $\mathrm{X}$ (Tabla 1) mostró que ambos tipos de sedimentos tuvieron este elemento en diferentes concentraciones. El $\mathrm{Mn}^{2+}$, al hacer interacción con el DAM, inició su solubilización hacia las fases líquidas. Como los valores del pH final de las soluciones son inferiores a lo que el $\mathrm{Mn}^{2+}$ necesita para poder precipitar $(\mathrm{pH} \geq 9)$, la mayor parte de este elemento permaneció en forma disuelta, en especial en los tratamientos con caliza (Figura 4D).

\subsection{LÍMITES MÁXIMOS PERMISIBLES}

Como es de interés en este trabajo conocer la eficiencia de los carbonatos pedogénicos en el tratamiento del DAM, se compararon los resultados obtenidos de las pruebas en lote con los límites máximos permisibles (LMP) de la NOM-001-SEMARNAT-1996, que establece los LMP de contaminantes en las descargas de aguas residuales en aguas y bienes nacionales.

La comparativa entre los EPT y los LMP se muestra en la Figura 4E. El Ni y el Cu son elementos que no presentaron problemas de contaminación después de las pruebas, ya que los resultados demuestran que las concentraciones se encontraron por debajo de los límites de detección, por lo que ninguna de las relaciones de CT y CS exceden los LMP.

La precipitación Gd resultó efectiva en la relación 1:2.5 de CT y C.S, por lo que no excede los LMP. Por el contrario, hay concentraciones disueltas en la relación 1:20, que se compararon con los LMP con un valor de promedio diario (PD) de $0.05 \mathrm{mg} / \mathrm{L}$ y un promedio mensual (PM) de $0.1 \mathrm{mg} / \mathrm{L}$. Se considera que esta relación se encuentra dentro del parámetro mensual normal, por lo que se concluye que su uso no genera un problema de contaminación.

Los LMP del Zn contemplan un valor de PD de 10 mg/L y un PM de 20 mg/L. Los resultados en ambas relaciones de CT y CS presentaron concentraciones disueltas pero se encuentran por debajo de los LMP. Por lo que el Zn no presenta problema de contaminación.

Por otro lado, Fe, $\mathrm{Al}$ y $\mathrm{Mn}$ son elementos considerados nocivos para la salud humana, pero no están contemplados por la NOM-001SEMARNAT-1996. Sin embargo, aparecen en la NOM-127-SSA1-1994, que establece los LMP para la calidad de agua, uso y consumo humano. Los tratamientos de CT y CS de ambas relaciones presentaron buena capacidad en precipitar Fe y 
$\mathrm{Al}$, por lo que no rebasan los LMP de la NOM127 que establece valores no mayores a $\mathrm{Fe}=0.3$ $\mathrm{mg} / \mathrm{L} \mathrm{y} \mathrm{Al}=0.2 \mathrm{mg} / \mathrm{L}$.

El Mn es un elemento liberado en estas pruebas, se compararon los resultados con los LMP que sugieren un valor de $\mathrm{Mn}=0.15 \mathrm{mg} / \mathrm{L}$. Como ambas relaciones de CT y CS en este estudio mostraron valores superiores de los LMP, sobre todo en CT, se considera necesario un tratamiento adicional como la oxidación-filtración, intercambio iónico, etc., antes de su uso de acuerdo a la norma (NOM-127). Pero, puesto que todo ellos son considerados procesos activos de alto costo, se sugieren tratamientos pasivos adicionales como los humedales anaerobios para el control del $\mathrm{Mn}$ (López et al., 2002).

\section{Conclusiones}

A partir de los resultados obtenidos se llegan las siguientes conclusiones:

- La capacidad de neutralizar el drenaje ácido de mina fue efectiva tanto en el edafosedimento como en la caliza.

- El edafosedimento tuvo la misma capacidad que la caliza para precipitar los mismos elementos potencialmente tóxicos.

- El tratamiento CS relación 1.2:5 tiene mayor probabilidad de formar sales por los altos contenidos de $\mathrm{Na}^{+}$.

- Por ello, la relación 1:20 resulta ser la más viable en el tratamiento del DAM; primero porque no hay problemas de salinidad y segundo porque se tiene la misma efectividad al tratar mayor volumen de líquido.

Se considera necesario continuar con pruebas por medio de columnas para conocer los tiempos útiles del edafosedimento y evaluar el control del Mn que se libera durante las pruebas.

\section{Agradecimientos}

Este trabajo agradece la valiosa contribución y colaboración de las siguientes personas: Dra. Teresa Pi I Puig del al laboratorio de Difracción de Rayos X por el apoyo en las difracciones de rayos X; a los técnicos Fabiola Vega, Inés Ramos y Gerardo Martínez del laboratorio de Geoquímica Ambiental por el apoyo en campo y trabajo de laboratorio. Estos Laboratorios pertenecen al Laboratorio Nacional de Geoquímica y Mineralogía (LANGEM), Instituto de Geología, UNAM. También se agradece el apoyo al técnico Jaime Díaz del taller de Laminación de Suelos por el asesoramiento en la elaboración e interpretación de las láminas delgadas.

\section{Referencias}

Akcil, A., Koldas, S., 2006, Acid mine drainage (AMD): causes, treatment and case studies: Journal of Cleaner Production, 14, 1139-1145. https://doi.org/10.1016/j.jclepro.2004.09.006

Asta Andrés, M.P., Acero Salazar, P., Auqué Sanz, L.F., Gimeno Serrano, M.J., Gómez Jiménez, J.B., 2011, Procesos geoquímicos en aguas ácidas por meteorización de sulfuros: Boletín Geológico y Minero, 2, 259-272.

Baird, C., 2001, Química ambiental: Barcelona, Editorial Reverté, 593 p.

Boul, S. W., Southard, R. J., Graham, R. C., McDaniel, P. A., 2011, Soil genesis and classification: Hoboken, Wiley-Blackwell, 531 p. https://doi.org/10.1002/9780470960622

Corrales-Pérez, D., Romero, F. M., 2013, Evaluación de la peligrosidad de jales de zonas mineras de Nicaragua y México y alternativas de solución: Boletín de la Sociedad Geológica Mexicana, 65(3), 427-446. https://doi.org/10.18268/ bsgm2013v65n3al 
Cravotta III, C. A., Kay, T. M., 1999, Limestone drains to increase $\mathrm{pH}$ and remove dissolved metals from acidic mine drainage: Applied Geochemistry, 14(5), 581-606. https://doi. org/10.1016/s0883-2927(98)00066-3

Gisbert, B. J., Ibáñez, A. S., 2008, Génesis de Suelos: Valencia, Universidad Politécnica de Valencia, Departamento de Producción Vegetal, $221 \mathrm{p}$.

Hammarstrom, J. M., Sibrell, P. L., Belkin, H. E., 2003, Characterization of limestone reacted with acid-mine drainage in a pulsed limestone bed treatment system at the Friendship Hill National Historical site, Pennsylvania, USA: Applied Geochemistry, 18(11), 1705-1721. https://doi.org/10.1016/ s0883-2927(03)00105-7

Kalin, M., Fyson, A., Wheeler, W. N., 2006, The chemistry of conventional and alternative treatment systems for the neutralization of acid mine drainage: Science of the Total Environment, 366, 395-408. https://doi. org/10.1016/j.scitotenv.2005.11.015

Krasilnikov, P., Gutiérrez-Castorena, M. C., Ahrens, R. J., Cruz-Gaistardo, C. O., Sedov, S., Solleiro-Rebolledo, E., 2013, The Soils of Mexico: World Soils Book Series, Springer. New York, NY, USA. 181 p. https://doi. org/10.1007/978-94-007-5660-1

López, P. E., Aduvire, O., Barenitto, D., 2002, Tratamientos pasivos de drenajes ácidos de mina: estado actual y perspectivas de fututo: Boletín Geológico y Minero, 1, 3-21.

Morin, K. A., Hutt, N. M., 1997, Environmental Geochemistry of minesite drainage: Practical theory and case studies: MDAG Publishing, 45-62.

Offeddu, F. G., Cama, J., Soler, J. M., Dávila, G., McDowell, A., Craciunescu, T., Tiseanu, I., 2015, Processes affecting the efficiency of limestone in passive treatments for AMD: column experiments: Journal of Environmental Chemical Engineering, 3(1), 304-316. https://doi.org/10.1016/j. jece.2014.10.013
Romero, F. M., Armienta, M. A., Gutierrez, M. E., Villaseñor G., 2008, Factores geológicos y climáticos que determinan la peligrosidad y el impacto ambiental de los jales mineros: Revista Internacional de Contaminación Ambiental, 2, 43-54.

Romero, F. M., Núñez, L., Gutiérrez, M. E., Armienta, M. A., Ceniceros-Gómez, A. E., 2011, Evaluation of the potential of indigenous calcareous shale for neutralization and removal of arsenic and heavy metals from acid mine drainage in the Taxco mining area, Mexico: Archives of Environmental Contamination and Toxicology, 60(2), 191-203. https://doi. org/10.1007/s00244-010-9544-z

Santomartino, S., Webb, J. A., 2007, Estimating the longevity of limestone drains in treating acid mine drainage containing high concentrations of iron: Applied Geochemistry, 22(11), 2344-2361. https://doi.org/10.1016/j. apgeochem.2007.04.020

Secretaria de Medio Ambiente y Recursos Naturales (SEMARNAT), 1996, Norma Oficial Mexicana que establece los límites máximos permisibles de contaminantes en las descargas de aguas residuales en aguas y bienes nacionales (NOM001-SERMARNAT-1996): México, Diario Oficial de la Federación, 24 de diciembre de 1996.

Secretaria de Medio Ambiente y Recursos Naturales (SEMARNAT), 2003, Norma Oficial Mexicana que establece el procedimiento para caracterizar los jales, así como las especificaciones y criterios para la caracterización y preparación del sitio, proyecto, construcción, operación y post-operación de presas de jales (NOM-141SEMARNAT-2003): México, Diario Oficial de la Federación, 17 de septiembre de 2003.

Secretaria de Salud ambiental (SSA) 1994, Norma Oficial Mexicana que establece "Salud ambiental, agua para uso y consumo humano límites permisibles de calidad y tratamientos a que debe someterse el agua para su potabilización"

(NOM-127-SSA1-1994): México, 22 de noviembre del 2000. 
Tack, F. M., Callewaert, W. J. J., Verloo, M. G., 1996, Metal solubility as a function of $\mathrm{pH}$ in a contaminated, dredged sediment affected by oxidation: Environmental Pollution, 91(2), 199-208. https://doi. org/10.1016/0269-7491(95)00049-6

Taylor, J., Pape, S., Murphy, N., 2005, A summary of passive and active treatment technologies for acid and metalliferous drainage (AMD). Fifth Australian Workshop on acid drainage: Australia, Earth Systems, 49 p.

Udayabhanu, S. G., Prasad, B., 2010, Studies on environmental impact of acid mine drainage generation and its treatment: an appraisal: Indian Journal of Environmental Protection, 30 (11), 953-957.
United States Environmental Protection Agency (U. S. EPA), 1998, Permeable reactive barrier technologies for contaminant remediation: Washington DC, U. S. Environmental Protection Agency, $102 \mathrm{p}$.

United States Environmental Protection Agency (U. S. EPA), 2014, Reference Guide to treatment technologies for mining-influenced water: Washington DC, U. S. Environmental Protection Agency, Office of Superfund Remediation and Technology Innovation, $94 \mathrm{p}$.

Valente, T. M., Leal-Gómez., 2009, Occurrence, properties and pollution potential of environmental minerals in acid mine drainage: Science of the Total Environment, 407(3), 1135-1152. https://doi.org/10.1016/j. scitotenv.2008.09.050 